\title{
Locating the QCD critical endpoint through finite-size scaling
}

\author{
N. G. Antoniou, ${ }^{*}$ F. K. Diakonos, ${ }^{\dagger}$ X. N. Maintas, ${ }^{\ddagger}$ and C. E. Tsagkarakis ${ }^{\S}$ \\ Faculty of Physics, University of Athens, GR-15784 Athens, Greece
}

(Received 25 May 2017; revised manuscript received 3 August 2017; published 13 February 2018)

\begin{abstract}
Considering the 3D Ising universality class of the QCD critical endpoint, we use a universal effective action for the description of the baryon-number density fluctuations around the critical region. Calculating the baryon-number multiplicity moments and determining their scaling with system's size, we show that the critical region is very narrow in the direction of the baryon chemical potential $\mu$ and wider in the temperature $(T)$ direction. In this context, published experimental results on local proton densityfluctuation measurements, obtained by intermittency analysis in transverse momentum space in NA49 central $A+A$ collisions at $\sqrt{s_{N N}}=17.2 \mathrm{GeV}(A=\mathrm{C}, \mathrm{Si}, \mathrm{Pb})$, restrict significantly the location $\left(\mu_{c}, T_{c}\right)$ of the QCD critical endpoint. The main constraint is provided by the freeze-out chemical potential of the $\mathrm{Si}+\mathrm{Si}$ system, which shows nonconventional baryon density fluctuations. An indicative solution, ignoring experimental uncertainties, is $119 \mathrm{MeV} \leq T_{c} \leq 162 \mathrm{MeV}, 252 \mathrm{MeV} \leq \mu_{c} \leq 258 \mathrm{MeV}$.
\end{abstract}

DOI: 10.1103/PhysRevD.97.034015

\section{INTRODUCTION}

The search for the QCD critical endpoint (CEP), remnant of the chiral symmetry breaking, at a finite baryon density and high temperature, is the main task in contemporary relativistic ion collision experiments [1,2]. Fluctuation analysis with global [3,4] and local measures [5] is the basic tool to achieve this goal. Up to now, an indication of such nonconventional fluctuations, which can be related to the CEP, has been observed in the freeze-out state of $\mathrm{Si}+\mathrm{Si}$ central collisions at the NA49 Super Proton Synchrotron (SPS) experiment with beam energy $\sqrt{s_{N N}}=$ 17.2 GeV [6]. However, the strong background and the poor statistics in the corresponding data set did not allow for convincing statements concerning the existence and the location of the CEP. Similarly, in Relativistic Heavy Ion Collider (RHIC) beam energy scan (BES)-I program, a nonmonotonic behavior of $\kappa \sigma^{2}$ (kurtosis times the variance) for net-proton distribution, compatible with theoretical proposals [7], was observed [4,8], but conclusive evidence for the location of the critical point is still pending, so its experimental hunt continues. From the theoretical side, the efforts are focused on lattice QCD calculations at a finite

\footnotetext{
*nantonio@phys.uoa.gr

†fdiakono@phys.uoa.gr

*xmaintas@phys.uoa.gr

¿ctsagkarakis@phys.uoa.gr
}

Published by the American Physical Society under the terms of the Creative Commons Attribution 4.0 International license. Further distribution of this work must maintain attribution to the author(s) and the published article's title, journal citation, and DOI. Funded by SCOAP. chemical potential in order to obtain the QCD phase diagram from first principles and predict the location of the CEP. Unfortunately, the lattice results obtained until now depend strongly on the method used to handle the well-known sign problem, and they do not converge to a well-defined critical chemical potential value $[9,10]$. Therefore, a first-principle prediction of the QCD CEP location, the holy grail of the physics of strongly interacting matter in our times, is still missing.

In the present work, we will make an effort to estimate the QCD CEP location employing an appropriate effective action for the thermodynamic description of the baryonic fluid around the critical region. To this end, we will assume that CEP belongs to the 3D Ising universality class, a hypothesis which is strongly supported by several theoretical works [11-15]. In this context, a universal effective action, found on the basis of a Monte Carlo simulation of the 3D Ising system in an external field [16], is an appropriate tool for the formulation of the QCD critical properties. In Sec. II, we adapt this action to the conditions implied by the formation of the critical fluid with a conserved baryon number in $A+A$ collisions of $\mathrm{QCD}$ matter introducing the Ising-QCD partition function to describe the associated thermal properties. Based on this partition function, in Sec. III, we calculate the moments of the baryon-number density and explore their finite-size scaling properties. We demonstrate that the Ising-QCD partition function reproduces consistently the finite-size scaling behavior expected for the 3D Ising universality class. In Sec. IV, we employ experimental data for the freeze-out parameters (chemical potential $\mu$ and temperature $T$ ) of the fireball formed in $A+A$ collisions in the NA49 experiment at CERN SPS with the highest beam 
energy $(\sqrt{s}=17 \mathrm{GeV})$ [17] as well as the result of the recent $\phi_{2}$ measurement in "Si" $+\mathrm{Si}$ collisions at the same experiment [6] to estimate the size of the finite-size scaling region. To achieve this, we first show that finite-size scaling in configuration space leads to a critical intermittency effect in transverse momentum space [5]. As a consequence the associated intermittency index $\phi_{2}$ is determined by the corresponding finite-size scaling exponent of the mean baryon number. Utilizing this relationship, we demonstrate the predictive power of an accurate measurement of $\phi_{2}$ combined with accurate estimates of $(\mu, T)$ for a system expected to freeze out within the finite-size scaling region. Thus, ignoring in our treatment experimental errors, we obtain an indicative solution for the location of the QCD CEP in the $(\mu, T)$ plane. Finally, in Sec. V, we present our concluding remarks.

\section{ISING-QCD PARTITION FUNCTION}

Introducing a dimensionless scalar field $\phi=\beta_{c}^{3} n_{b}$ (order parameter) with $\beta_{c}=1 / k_{B} T_{c}$ and $n_{b}$ the baryon-number density, the 3D Ising effective action, after integrating out short-wavelength fluctuations of $\phi$, is written, in the neighborhood of $T \approx T_{c}$ and $\mu \approx \mu_{c}$, as follows:

$$
\begin{aligned}
S_{\mathrm{eff}} & =\int_{V} d^{3} \hat{\mathbf{x}}\left[\frac{1}{2}|\hat{\nabla} \phi|^{2}+U(\phi)-\hat{h} \phi\right], \\
U(\phi) & =\frac{1}{2} \hat{m}^{2} \phi^{2}+\hat{m} g_{4} \phi^{4}+g_{6} \phi^{6} .
\end{aligned}
$$

In Eq. (1), the variables with a "hat" are dimensionless: $\hat{x}_{i}=x_{i} \beta_{c}^{-1}, \hat{m}=\beta_{c} m\left(m=\xi^{-1}, \xi\right.$ being the correlation length), $\hat{h}=\left(\mu-\mu_{c}\right) \beta_{c}$ (ordering field), and $g_{4}=0.97 \pm$ $0.02, g_{6}=2.05 \pm 0.15$ are universal dimensionless couplings [16]. At this point, it is of interest to note that the Ising-QCD description through the potential (1) is a valid approximation close to the critical point where there is no fundamental distinction between the two phases (quark matter versus hadronic matter in our case). As a result, the freeze-out states in nuclear collisions may occupy, in this restricted area of the phase diagram, both sectors of the temperature $\left(T \gtrsim T_{c}\right.$ or $\left.T \lesssim T_{c}\right)$ and not necessarily the conventional one $T \lesssim T_{c}$. In fact, since the correlation length differs as we approach from above or below the critical temperature $T_{c}$, the parameter $m$ is defined with two branches: $m=m_{+}=\xi_{+}^{-1}\left|\frac{T-T_{c}}{T_{c}}\right|^{\nu}$ for $T>T_{c}$ and $m=$ $m_{-}=\xi_{-}^{-1}\left|\frac{T_{c}-T}{T_{c}}\right|^{\nu}$ for $T<T_{c}$, with a universal amplitude ratio $\frac{\xi_{+}}{\xi_{-}} \approx 2$ [18] and critical exponent $\nu \approx 2 / 3$ [19] in the 3D Ising universality class.

The partition function, on the basis of Eq. (1), is written schematically:

$$
\mathcal{Z}=\sum_{\{\phi\}} \exp \left(-S_{\text {eff }}\right)
$$

Because of baryon-number conservation, $n_{b}$ is a slow mode of the order parameter at macroscopic scales (long-wavelength fluctuations). Thus, the sum in Eq. (2) is dominated by approximately constant configurations $(\hat{\nabla} \phi=0)$. Employing this ensemble to saturate the sum in Eq. (2), a discrete variable $\phi_{N}=\beta_{c}^{3} \frac{N}{V}$ is introduced in the summation ( $N$ is the proton multiplicity and $V$ the volume of the finite system) leading to the expansion:

$$
\mathcal{Z}=\sum_{N=0}^{M} \zeta^{N} \exp \left[-\frac{1}{2} \hat{m}^{2} \frac{N^{2}}{M}-g_{4} \hat{m} \frac{N^{4}}{M^{3}}-g_{6} \frac{N^{6}}{M^{5}}\right],
$$

where $\zeta=\exp \left(\frac{\mu-\mu_{c}}{k_{B} T_{c}}\right), M=\frac{V}{\beta_{c}^{3}}, \hat{m}=\beta_{c} \xi^{-1}=\beta_{c} \xi_{ \pm}^{-1}|t|^{\nu}$, with $t=\frac{T-T_{c}}{T_{c}}$ the reduced temperature, $\beta_{c} \xi_{+}^{-1}=1\left(T>T_{c}\right)$, and $\beta_{c} \xi_{-}^{-1}=2\left(T<T_{c}\right)$. Using the partition function (3), it is straightforward to calculate the baryon-number distribution moments:

$$
\left\langle N^{k}\right\rangle=\frac{1}{\mathcal{Z}} \sum_{N=0}^{M} N^{k} \zeta^{N} \exp \left[-\frac{1}{2} \hat{m}^{2} \frac{N^{2}}{M}-g_{4} \hat{m} \frac{N^{4}}{M^{3}}-g_{6} \frac{N^{6}}{M^{5}}\right]
$$

with $k=1,2, \ldots$ and explore their scaling behavior with the system's size $M$ around the critical region. At the critical point $\zeta_{c}=1\left(\mu=\mu_{c}\right), \hat{m}_{c}=0\left(T=T_{c}\right)$, these moments obey the scaling law:

$$
\left\langle N^{k}\right\rangle \sim M^{k q} ; \quad q=d_{F} / d, \quad k=1,2, \ldots,
$$

where $d$ is the embedding dimension of the considered system and $d_{F}$ the fractal dimension related to the critical fluctuations of the baryon density [20].

\section{FINITE-SIZE SCALING IN THE ISING-QCD MODEL}

It is important to demonstrate that the effective action (1) is fully compatible with the theory of finite-size scaling within the 3D Ising universality class. In fact, summarizing the general aspects of this theory [21,22] for the basic thermodynamic quantities (density $n$, specific heat $c$, and susceptibility $\chi$ ) of a critical system, we obtain the behavior for large but finite volume $V$ (with $V=M V_{0}$ ):

$$
n \sim V^{-\beta / \nu d} ; \quad c \sim V^{\alpha / \nu d} ; \quad \chi \sim V^{\gamma / \nu d}
$$

at the critical point $t=0, \zeta=1$. The exponents $(\alpha, \beta, \gamma)$ are linked to the scaling power laws of the infinite system: $n \sim t^{\beta}, c \sim t^{-\alpha}$, and $\chi \sim t^{-\gamma}$. In the case of the Ising-QCD system, we have found on the basis of the partition function (3) $n_{b} \sim V^{q-1}$ with $q=\frac{d_{F}}{d}, d_{F}=d-\frac{\beta}{\nu}$ leading to the behavior $n_{b} \sim V^{-\beta / \nu d}$. 


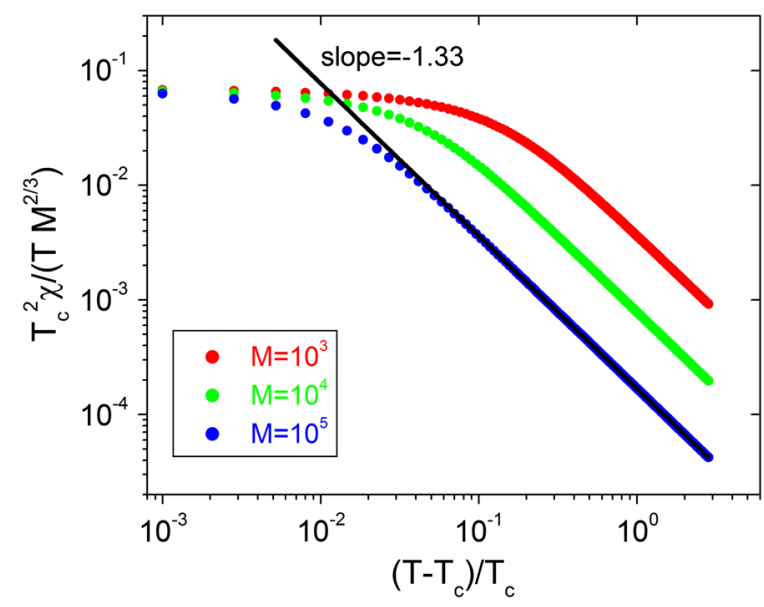

FIG. 1. The baryon-number susceptibility scaled by $T M^{2 / 3} / T_{c}^{2}$ versus $\left(T-T_{c}\right) / T_{c}$ plotted in the double logarithmic scale for three different values of the size $M: 10^{3}$ (red circles), $10^{4}$ (green circles), and $10^{5}$ (blue circles). The slope in the linear fit determines the critical exponent $\gamma$. All sets converge to the same value for the scaled susceptibility for $T \rightarrow T_{c}$, verifying the finitesize scaling relation (6).

For the specific heat, we have $c=-\frac{T^{3}}{V} \frac{\partial^{2} \ln \mathcal{Z}}{\partial T^{2}}$ leading, through differentiation, to the components $c_{1} \sim \frac{1}{V}\left(\frac{1}{\mathcal{Z}} \frac{\partial^{2} \mathcal{Z}}{\partial^{2} t}\right)$ and $c_{2} \sim \frac{1}{V}\left(\frac{1}{\mathcal{Z}} \frac{\partial \mathcal{Z}}{\partial t}\right)^{2}$. In the limit $t \rightarrow 0$, we obtain the leading behavior: $c_{1} \sim \frac{t^{\nu-2}}{V^{4}}\left\langle N^{4}\right\rangle, c_{2} \sim \frac{t^{2 \nu-2}}{V^{7}}\left\langle N^{8}\right\rangle$, and, on the basis of Eq. (5), we may write

$$
c_{1} \sim t^{\nu-2} V^{4(q-1)} ; \quad c_{2} \sim t^{2 \nu-2} V^{8 q-7}
$$

with $q=\frac{5}{6}$ for the 3D Ising case. Approaching the regime of finite-size scaling, the correlation length grows with the size of the system, $\xi \sim V^{1 / 3}$, and the appropriate path in Eq. (7) towards this regime is $t \sim V^{-1 / 3 \nu}$. As a result, both components $c_{1}$ and $c_{2}$ have the same scaling behavior $c_{1,2} \sim V^{(2-3 \nu) / 3 \nu}$ leading to the finite-size scaling law for the specific heat:

$c \sim V^{\alpha / \nu d} ; \quad d=3, \quad \alpha=2-\nu d \quad$ (Josephson law).

Finally, for the susceptibility, we employ fluctuationdissipation relations:

$\chi=\frac{T}{T_{c}^{2} V}\left\langle(\delta N)^{2}\right\rangle ; \quad\left\langle(\delta N)^{2}\right\rangle=\zeta \frac{\partial}{\partial \zeta}\left(\zeta \frac{\partial \log \mathcal{Z}}{\partial \zeta}\right)$

at the critical point, where $\left\langle N^{k}\right\rangle \sim M^{k q}$. As a result, $\left\langle(\delta N)^{2}\right\rangle \sim V^{2 q}$ and $\chi \sim V^{2 q-1}$ with $2 q-1=\frac{\gamma}{\nu d}$, recovering the finite-size scaling behavior $\chi \sim V^{\gamma / \nu d}$ in Eq. (6).

For completeness, we have also studied the behavior of the infinite system $(M \rightarrow \infty)$ in the limit $T \rightarrow T_{c}$, where we expect $\chi(T) \sim\left|T-T_{c}\right|^{-\gamma}$ with $\gamma \approx \frac{4}{3}$ in the 3D Ising universality class. In Fig. 1, plotting $T_{c}^{2} \chi(T) /\left(T M^{2 q-1}\right)$ for various values of $M$, calculated numerically through the partition function (3), we clearly show the validity of both scaling laws, for finite and infinite systems.

Summarizing the discussion in this section, we have argued that the effective action (1) captures correctly all the scaling aspects of the critical behavior with the appropriate Ising exponents: $\alpha \approx 0, \beta \approx \frac{1}{3}, \gamma \approx \frac{4}{3}, \delta \approx 5$, and $\nu \approx \frac{2}{3}$. In particular, we have demonstrated that the fractal fluctuations $\langle N\rangle \sim V^{d_{F} / d}$, discussed in the previous section, are linked to the finite-size scaling of the system $\left(n \sim V^{-\beta / \nu d}\right)$, which is the origin of critical fluctuations in a finite system.

\section{LOCATING THE QCD CEP WITH FINITE-SIZE SCALING}

Our strategy to determine the location of the QCD CEP is the following. First, we will estimate the size of the critical region based on the scaling behavior of $\langle N\rangle$ with the system's size $M$. Then, using the published NA49 results on proton intermittency analysis in central $A+A$ collisions at $\sqrt{s_{N N}}=17.2 \mathrm{GeV}$ [6], we will constrain the location of the CEP. For the second step, it is crucial that the critical exponent $q$ in Eq. (5) is directly related to the intermittency index $\phi_{2}$ measured in the proton intermittency analysis. Let us start with the estimation of the critical region. We determine the dependence of $\langle N\rangle$ on $M$ for different values of $\zeta$ and $\hat{m}$. Exactly at the critical point $\left(\zeta_{c}, \hat{m}_{c}\right)=(1,0)$, the critical exponent $q$ attains the value 5/6 for the 3D Ising universality class $(\delta=5)$ extracted from the representation (3). A direct calculation of $\langle N\rangle$ as a function of $M$ from the partition function in Eq. (3) shows that departing slightly from the critical point leads to a behavior $\langle N\rangle \sim M^{\tilde{q}}$ for $M \gg 1$ with $\tilde{q} \neq q$. Outside the critical region, $\tilde{q}=1$. Varying $\hat{m}$ and $\zeta$, we may also enter to the $\phi^{4}$-dominance region when the last term in the effective action (1) becomes suppressed with respect to the other two effective potential terms. In that case, $\tilde{q}=3 / 4$ (mean field universality class, $\delta=3$ ), and the information of the 3D Ising critical exponent $q$ is again lost. Thus, we consider as a critical region of the QCD CEP the domain in the $(\zeta, \hat{m})$ or, equivalently, the $(\ln \zeta, t)$ plane for which

$$
\langle N\rangle \sim M^{\tilde{q}}, \quad 3 / 4<\tilde{q}<1,
$$

holds.

Since the critical region depends, in general, on the size $M$, a supplementary constraint that the correlation length is greater than the linear size of the system, compatible with the finite-size scaling theory $[21,23]$, is certainly needed. To keep contact with $M$ values realistic for the size of the fireball produced in relativistic ion collisions, we explore the validity of the scaling law (6) for $20<M<700$. This estimated range of $M$ values contains all sizes of nuclei 

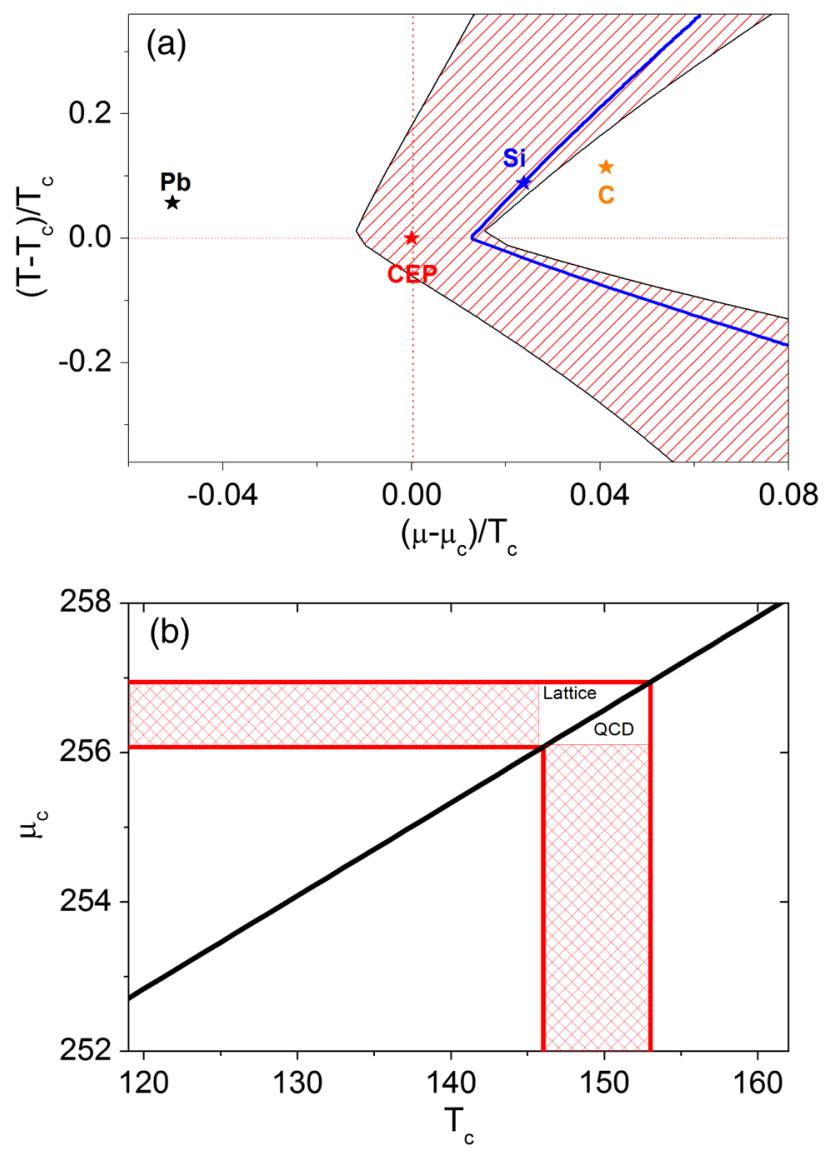

FIG. 2. (a) The critical region (red shaded area) in the plane $\left(\mu-\mu_{c}\right) / T_{c},\left(T-T_{c}\right) / T_{c}$ where the scaling law $\langle N\rangle \sim M^{\tilde{q}}$ with $\tilde{q} \in(0.75,1)$ holds. The blue line is the line for which $\tilde{q}=0.96$. The freeze-out states for central $A+A$ collisions in the NA49 experiment at maximum SPS energy according to Ref. [17] are given by the stars: $A=\mathrm{Pb}$ (black), $A=\mathrm{Si}$ (blue), and $A=\mathrm{C}$ (orange). (b) The line describes the possible pairs $\left(T_{c}, \mu_{c}\right)$ compatible with the finite-size analysis in the current work. The red patterned region is determined employing the different lattice QCD results for the critical temperature $T_{c}[9,10]$.

ranging from $\mathrm{Be}\left(R_{\mathrm{Be}} \approx 2.6 \mathrm{fm}\right)$ to $\mathrm{Pb}\left(R_{\mathrm{Pb}} \approx 7.5 \mathrm{fm}\right)$, assuming $T_{c} \approx 150 \mathrm{MeV}$. In Fig. 2(a), the red shaded area denotes the critical region, i.e., the domain in $(\ln \zeta, t)$ plane for which the above constraints hold. We observe that the critical region is quite extended in the $t$ direction but it is very narrow in the $\ln \zeta$ direction. This is a crucial property restricting the location of the CEP. The blue line in Fig. 2(a) is the location of the $(\ln \zeta, t)$ pairs, which lead to a scaling of $\langle N\rangle$ with $\tilde{q}=0.96$. The origin of this value of the exponent $\tilde{q}$ is explained in the following: It can be proven that $\tilde{q}$ is, in fact, the value of the intermittency index $\phi_{2}$ in transverse momentum space. The proof is based on the following simple rules of fractal geometry: Owing to finitesize scaling, the two-particle correlator, at scales close to the correlation length, $|\delta \mathbf{x}| \sim \xi$, has a power-law behavior of the form $\left\langle n_{b}(\delta \mathbf{x}) n_{b}(\mathbf{0})\right\rangle \sim|\delta \mathbf{x}|^{d_{F}-d}$ which reveals fluctuations of the critical system at large distances. In transverse space and in the central region of a nuclear collision $(d=3)$, where longitudinal and transverse geometries are disentangled [5,24], the fractal dimension is $d_{F}^{(2)}=\frac{2}{3} d_{F}$. Finally, in momentum space, the density-density correlation $\left\langle n_{b}\left(\delta \mathbf{q}_{\perp}\right) n_{b}(\mathbf{0})\right\rangle$ at small scales $\left|\delta \mathbf{q}_{\perp}\right| \sim \xi^{-1}$, reflecting the fluctuations at large distances (in transverse plane), satisfies a power law which gives rise to $2 \mathrm{D}$ intermittency as follows:

$$
\begin{aligned}
\left\langle n_{b}\left(\delta \mathbf{q}_{\perp}\right) n_{b}(\mathbf{0})\right\rangle & \sim\left|\delta \mathbf{q}_{\perp}\right|^{-(2 / 3) d_{F}}, \\
\text { (2nd factorial moment) } & \sim(\text { size of 2D bin })^{-\phi_{2}^{(c r)}}
\end{aligned}
$$

with $\phi_{2}^{(c r)}=\frac{d_{F}}{3}$. As a result, the measurable exponent $\phi_{2}$ in intermittency experiments is identified with the finite-size scaling exponent $\tilde{q}$.

Coming back to Fig. 2(a), according to the previous discussion, the mean value of the intermittency index $\phi_{2} \approx 0.96$ found in the SPS NA49-data analysis of the proton-density fluctuations in transverse momentum space for $\mathrm{Si}+\mathrm{Si}$ central collisions at $\sqrt{s_{N N}}=17.2 \mathrm{GeV}$ corresponds to $\tilde{q}=0.96$. Thus, the $\mathrm{Si}+\mathrm{Si}$ freeze-out state should lie on the blue line $(\tilde{q}=0.96)$ plotted in Fig. 2(a). Taking the freeze-out data of the fireball created in central collisions of $\mathrm{Si}+\mathrm{Si}$ at $\sqrt{s_{N N}}=17.2 \mathrm{GeV}$ to be $(\mu, T)=(260,162) \mathrm{MeV}$, as reported in Ref. [17], the condition of lying on the blue line provides a relation between $\mu_{c}$ and $T_{c}$. This relation, determining all possible values for the critical parameters of the QCD CEP compatible with our analysis and with the intermittency results in Ref. [6], is presented graphically in Fig. 2(b). The freezeout temperature of $\mathrm{Si}+\mathrm{Si}$ sets an upper bound on the critical temperature $T_{c}<162 \mathrm{MeV}$. A lower bound on $T_{c}$ is provided by the requirement that the correlation length $\xi$ is greater than the linear system size for the occurrence of critical fluctuations as stated above. For the smallest considered system, $\mathrm{Be}$, with a radius of $\approx 2.6 \mathrm{fm}$, we obtain the upper bound $|t|<0.36$, which covers also the case of Si and leads [Fig. 2(b)] to the lower bound on the critical temperature $T_{c}>119 \mathrm{MeV}$. Thus, in the plot in Fig. 2(b), we show only the allowed range $119 \mathrm{MeV}<T_{c}<162 \mathrm{MeV}$. We observe that the corresponding critical chemical potential domain is very narrow: $252 \mathrm{MeV}<\mu_{c}<258 \mathrm{MeV}$. The size of this domain is mainly due to the narrowness of the critical region along the $\ln \zeta$ direction for $T \approx T_{c}$, and it is not sensitive to the experimental input. In fact, using the available lattice QCD estimates of the critical temperature $T_{c} \approx 146 \mathrm{MeV}$ [10] and $T_{c} \approx 153 \mathrm{MeV}$ [9] as the borders of a critical zone [red shaded domain in Fig. 1(b)], we obtain a very narrow range for the critical chemical potential $256 \mathrm{MeV}<\mu_{c}<257 \mathrm{MeV}$. For completeness, we add in Fig. 2(a) the freeze-out states for the central collisions of the other two systems, $\mathrm{Pb}+\mathrm{Pb}$ and $\mathrm{C}+\mathrm{C}$, considered in the NA49 experiment (at $\sqrt{s_{N N}}=17.2 \mathrm{GeV}$ ). We clearly observe that these systems lie outside the critical (shaded) region, although their freeze-out chemical-potential values do not differ so much. The reason is the narrowness of 

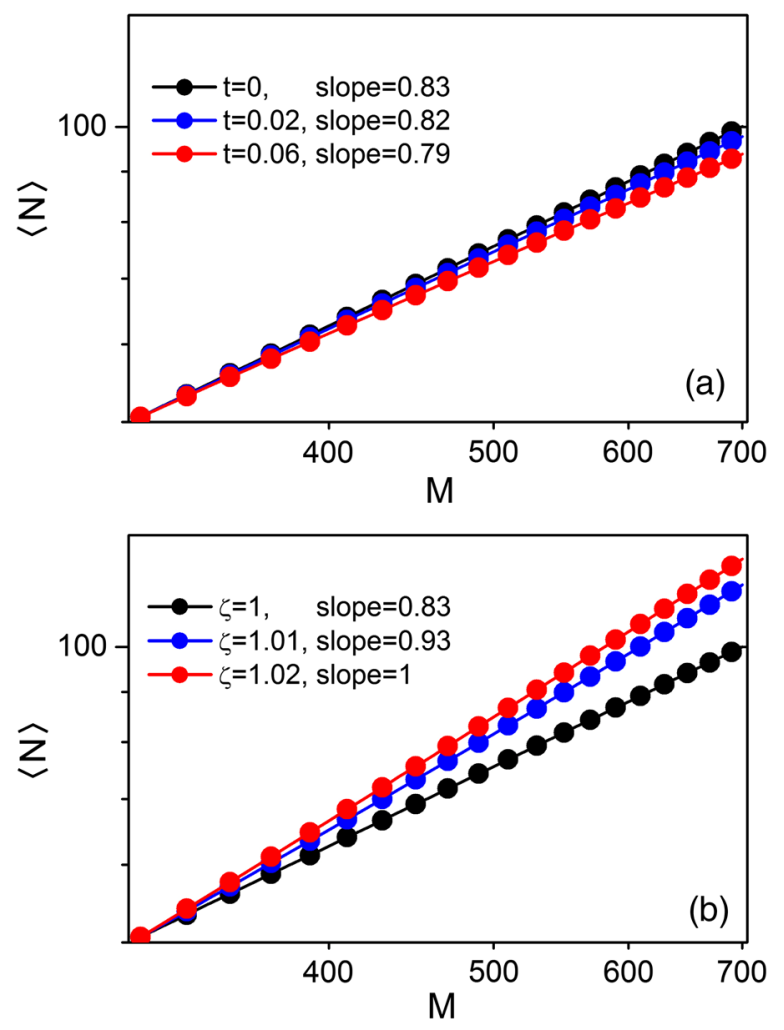

FIG. 3. (a) The first moment of the baryon-number density $\langle N\rangle$ as a function of $M$ for $t=0,0.02,0.06$ (corresponding to $\hat{m}=0$, $0.07,0.15$ ) and $\zeta=\zeta_{c}=1$. (b) The mean value $\langle N\rangle$ as a function of $M$ for $T=T_{c}(\hat{m}=0)$ and $\zeta=1,1.01,1.02$.

the critical region in the chemical potential direction which possesses a linear size of $4.5 \mathrm{MeV}$ for $T=T_{c}$ and $T_{c} \approx 150 \mathrm{MeV}$.

To illustrate in more detail how our strategy, leading to the critical region in Fig. 2(a), works in practice, we plot in Fig. 3, in the double logarithmic scale, the mean value $\langle N\rangle$ versus $M$ for various values of $t$ and $\zeta$. The plot focuses on the relevant region $310 \leq M \leq 700$. To facilitate the comparison, we have scaled all moments to a common value at $M=310$ using as a reference the black line $(t=0$ and $\zeta=1)$. In Fig. 3(a), we show $\langle N\rangle$ for $t=0,0.02,0.06$ and $\zeta=1$. We observe that, although $t$ increases by a factor of 3 -or, equivalently, $\hat{m}$ by a factor of 2 -, the corresponding slope change is relatively small. This slow change of the exponent $\tilde{q}$ with increasing $t$ (or $\hat{m}$ ) explains why the critical region in the $t$ (or $\hat{m}$ ) direction is wide. On the other hand, assuming $T=T_{c}$ and varying $\zeta$ across the real axis, we observe that the critical scaling goes over to the conventional behavior $\langle N\rangle \sim M$ quite rapidly. This behavior is in accordance with the plot of the critical region in Fig. 2(a), and it is clearly demonstrated in Fig. 3(b), where we plot in the double logarithmic scale $\langle N\rangle$ versus $M$ for three different $\zeta$ values $\left(1,1.01\right.$, and 1.02) and for $T=T_{c}$. Notice that in Fig. 3(a) the slope decreases as we depart from the critical (black) line, going over to the mean field behavior, while in Fig. 3(b) it increases, going over to the conventional behavior mentioned above. Furthermore, it is worth also to mention that the value of $\langle N\rangle$, obtained through Eq. (4), is a prediction for the total mean baryonnumber multiplicity in the critical freeze-out state, since there are no free parameters in the calculation. This information is shown by the black line in Fig. 3(a) [or Fig. 3(b)], which gives the mean baryon number as a function of the system's size $M$.

\section{CONCLUSIONS}

Concluding remarks are now in order. We have proposed the effective action (1) for the description of critical QCD fluctuations, very close to the critical point, in a region where the two phases (hadron and quark) are indistinguishable. It is inspired by the universal effective potential, simulating the 3D Ising system for $T>T_{c}$, found in Ref. [16]. In this Ising-QCD description, two fundamental critical indices $\left(q=\frac{5}{6}, \nu=\frac{2}{3}\right)$ are incorporated with their values dictated by the 3D Ising universality class. In fact, in Sec. III, we have shown that this description is compatible with all requirements of the scaling theory for finite and infinite critical systems within the above universality class. In Sec. IV, we have demonstrated on the basis of (1) that the critical region linked to the CEP is relatively wide along the temperature direction (of the order of $30 \mathrm{MeV}$ ) and very narrow along the chemical potential axis (of the order of $5 \mathrm{MeV}$ ). This property becomes instrumental in our effort to locate the critical point in the phase diagram. In fact, using published results on the intermittency analysis of proton-density fluctuations in the SPS NA49 experiment [6] and lattice QCD estimates of the critical temperature, it is possible to give a prediction for the location of the QCD CEP: $\left(\mu_{c}, T_{c}\right) \approx(256,150) \mathrm{MeV}$ as shown in Fig. 1(b). However, this is only an indicative solution, since we have ignored experimental uncertainties, underlining the need for high-precision experimental measurements of protonproton correlations (for the accurate determination of intermittency index $\phi_{2}$ ) and particle yields (for the accurate extraction of the corresponding freeze-out parameters). These very accurate measurements require high statistics as well as control of some spurious effects occurring in ion collision experiments like volume and temperature fluctuations, acceptance constraints, impact of centrality bin width, critical slowing down, influence of resonance decays, etc. Concerning intermittency analysis, the use of local fluctuation measures ensures insensitivity to acceptance and global (size and temperature) fluctuations. Furthermore, in the same context, resonance decays do not contribute to local power-law fluctuations, as shown in Ref. [6] employing the EPOS Monte Carlo generator for ion collisions. High statistics would also allow to reduce the width of the centrality bins avoiding the influence of related effects to the intermittency analysis. Finally, the approach presented here assumes that the freeze-out states produced in ion collisions are equilibrated, as supported also by the 
experimental data. Therefore, critical slowing down is not expected to have any impact on intermittency analysis. Thus, overall, the accurate measurement of $\phi_{2}$ in current ion collision experiments is a feasible task.

From the analysis above, one may draw conclusions about the most promising, crucial measurements in the experiments, currently in progress, at CERN and Brookhaven National Laboratory (BNL) (SPS-NA61 and RHIC-BES). It is suggestive from the constraints of the critical region in Fig. 2 that 2D intermittency [25] of net-proton density in transverse momentum space (in the central rapidity region) combined with chemical freeze-out measurements may capture the systems, for different energies $\left(\sqrt{s_{N N}}\right)$ and size of nuclei $(A)$, which freeze out very close to the critical point. To this end, we consider two classes of experiments with heavy (I) and medium or small size (II) nuclei.
(1) $\mathrm{Pb}+\mathrm{Pb}, \mathrm{Au}+\mathrm{Au}$.- The crucial energy range for these processes in the experiments at CERN $(\mathrm{Pb}+\mathrm{Pb})$ and $\mathrm{BNL}(\mathrm{Au}+\mathrm{Au})$, compatible with the requirements of the critical region (Fig. 2), is

(a) $\mathrm{Pb}+\mathrm{Pb}$ at $12.3 \mathrm{GeV}<\sqrt{s_{N N}}<17.2 \mathrm{GeV}$ (SPS-NA61) corresponding to lab-energies $80 \mathrm{AGeV}<E_{\mathrm{lab}}<158 \mathrm{AGeV}$ and

(ii) $\mathrm{Au}+\mathrm{Au}$ at $11.5 \mathrm{GeV}<\sqrt{s_{N N}}<14.5 \mathrm{GeV}$ (RHIC-BES).

(2) $\mathrm{Be}+\mathrm{Be}, \mathrm{Ar}+\mathrm{Sc}, \mathrm{Xe}+\mathrm{La}$.- The crucial measurements in these processes, regarding 2D intermittency, are in progress at the experiment SPS-NA61, at the highest SPS energy $\sqrt{s_{N N}}=17.2 \mathrm{GeV}$. To complete the picture, however, a detailed study of chemical freeze-out in these collisions is also needed.
[1] M. Gazdzicki (for the NA61 Collaboration), J. Phys. G 36, 064039 (2009); N. Antoniou et al. (NA61/SHINE Collaboration), Report No. CERN-SPSC-2006-034.

[2] M. M. Aggarwal et al. (STAR Collaboration), arXiv:1007 .2613; STAR Note No. 0598: BES-II white paper: http:// drupal.star.bnl.gov/STAR/starnotes/public/sn0598.

[3] M. A. Stephanov, Int. J. Mod. Phys. A 20, 4387 (2005); J. Phys. G 38, 124147 (2011).

[4] X. Luo and N. Xu, Nucl. Sci. Tech. 28, 112 (2017).

[5] N. G. Antoniou, F. K. Diakonos, A. S. Kapoyannis, and K. S. Kousouris, Phys. Rev. Lett. 97, 032002 (2006).

[6] T. Anticic et al., Eur. Phys. J. C 75, 587 (2015).

[7] M. A. Stephanov, Phys. Rev. Lett. 107, 052301 (2011).

[8] X. Luo, Nucl. Phys. A956, 75 (2016).

[9] Z. Fodor and S. Katz, J. High Energy Phys. 04 (2004) 050.

[10] R. V. Gavai and S. Gupta, Phys. Rev. D 78, 114503 (2008).

[11] S. Gavin, A. Gocksch, and R. D. Pisarski, Phys. Rev. D 49, R3079 (1994).

[12] M. Stephanov, K. Rajagopal, and E. Shuryak, Phys. Rev. Lett. 81, 4816 (1998).

[13] M. A. Halasz, A. D. Jackson, R. E. Shrock, M. A. Stephanov, and J. J. M. Verbaarschot, Phys. Rev. D 58, 096007 (1998).
[14] J. Berges and K. Rajagopal, Nucl. Phys. B538, 215 (1999).

[15] F. Karsch, E. Laermann, and Ch. Schmidt, Phys. Lett. B 520, 41 (2001).

[16] M. M. Tsypin, Phys. Rev. Lett. 73, 2015 (1994).

[17] F. Becattini, J. Manninen, and M. Gazdzicki, Phys. Rev. C 73, 044905 (2006).

[18] V. Privmam, P. C. Hohenberg, and A. Aharony, in Phase Transitions and Critical Phenomena, Vol. 14, edited by C. Domb and J. L. Lebowitz (Academic, New York, 1989).

[19] A. Pelissetto and E. Vicari, Phys. Rep. 368, 549 (2002).

[20] N. G. Antoniou, F. K. Diakonos, X. N. Maintas, and C. E. Tsagkarakis, Phys. Rev. E 95, 052145 (2017).

[21] H. Meyer-Ortmanns, Rev. Mod. Phys. 68, 473 (1996).

[22] E. S. Fraga, L. F. Palhares, and P. Sorensen, Phys. Rev. C 84, 011903 (2011).

[23] R. B. Stinchcombe, Order and Chaos in Nonlinear Physical Systems (Plenum, New York, 1988).

[24] N. G. Antoniou, N. Davis, and F. K. Diakonos, Phys. Rev. C 93, 014908 (2016).

[25] A. Bialas and R. Peschanski, Nucl. Phys. B273, 703 (1986); 308, 857 (1988). 